Zeitschriftenartikel:

Begutachtet

Redaktion und Begutachtung:

Nele Heise (iD)

Digital Media \& Communication

Researcher Hamburg

Nils Zurawski (DD

Universität Hamburg

Erhalten: 01. Dezember 2019

Akzeptiert: 29. September 2020

Publiziert: 15. Dezember 2020

Lizenz:

(C) Sven Preger

Dieses Werk steht unter einer Lizenz

Creative-Commons-Namensnennung 4.0

(CC-BY 4.0) International

(C) (i)

Datenverfügbarkeit:

Alle relevanten Daten befinden sich innerhalb der Veröffentlichung.

Interessenskonfliktstatement:

Die Autor:innen erklären, dass ihre Forschung ohne kommerzielle oder finanzielle Beziehungen durchgeführt wurde, die als potentielle

Interessenskonflikte ausgelegt werden können.

Empfohlene Zitierung:

Preger, S. (2020). Dokumentarisches Erzählen in Podcasts: Wenn Storytelling auf Journalismus trifft - Ein Praxisbericht. kommunikation@gesellschaft, 21(2). https://doi.org/10.15460/kommges. 2020.21.2.634

\section{Dokumentarisches Erzählen in Podcasts}

\author{
Wenn Storytelling auf Journalismus trifft - Ein Praxisbericht
}

\author{
Sven Preger ${ }^{\mathrm{a} *}$ (D) \\ a Journalist, Bonn \\ *Korrespondenz: sven.preger@hoerweiten.de
}

\begin{abstract}
Im journalistischen Kontext verlaufen zwei Entwicklungen parallel: Einerseits entstehen neue Podcast-Formate, die entweder komplett narrativ sind oder einzelne Storytelling-Techniken integrieren. Andererseits stößt Storytelling im journalistischen Kontext häufig auf Ablehnung. Dieser Praxisbericht klärt, woher diese Ablehnung kommt und was unter Storytelling in journalistischen Podcasts zu verstehen ist. Darauf aufbauend werden zentrale Storytelling-Techniken erläutert und auf ihre journalistische Tauglichkeit überprüft. Dazu gehören: Plot-Entwurf, szenisches Erzählen, Erzählhaltung, Einsatz von Nähe und Intimität sowie akustische Gestaltung. Abschließend wird ein Blick darauf geworfen, welchen Platz Storytelling in journalistischen Podcasts einnehmen kann und welche besondere journalistische Qualität Storytelling-Podcasts entfalten können.
\end{abstract}

Schlagworte: Storytelling, Podcast, Plot, Erzählhaltung, Sounddesign 


\section{Warum Storytelling Journalist:innen irritiert}

Kaum etwas klingt so schön wie ein Moment echter Erkenntnis. Manchmal baut sie sich langsam auf und bricht dann als eine Mischung aus Frage und Ausruf hervor: „Dann kopieren die also das Falsche!?“ Diese Erkenntnis platzt regelrecht aus einer Seminarteilnehmerin hervor. Ihr knapper Satz bringt ein zentrales Problem in Sachen Storytelling und Podcast auf den Punkt.

Zur Einordung: Wir hatten im Workshop ,Storytelling für kurze Formen' zuvor zahlreiche handwerkliche Aspekte besprochen: fesselnde Einstiege, Spannungsaufbau in Kollegengesprächen sowie Interviewtechniken für Storys. Die Teilnehmer:innen hatten auch immer wieder grundsätzliche Fragen gestellt. Eine davon bekomme ich in den vergangenen Jahren immer wieder in Seminaren, auf Podien oder bei Formatentwicklungen gestellt. ${ }^{1}$ Diese Frage lautet dann häufig ungefähr so: Was lässt große Produktionen wie This American Life $^{2}$, Radiolab ${ }^{3}$, Invisibilia ${ }^{4}, S$-Town ${ }^{5}$ und Serial ${ }^{6}$, aber auch Terrible, thanks for asking $^{7}$, Love and Radio ${ }^{8}$ oder $99 \%$ Invisible ${ }^{9}$ lebendiger als viele deutschsprachige Podcasts klingen?

Besonders aufschlussreich finde ich dabei die Reaktionen auf diese Frage. Kaum gestellt, ist häufig ein gewisser Widerstand im Raum zu spüren, der verbalisiert dann so klingt: „Müssen wir denn alles so machen wie die Amerikaner?“ Oder: „Das ist eben ein kultureller Unterschied!“ Dabei gibt es mittlerweile etliche herausragende deutschsprachige Produktionen, dazu gehören: das Audible-Format Der Moment ${ }^{10}, 180$ Grad: Geschichten gegen den Hass $^{11}$ (NDR Info), 4 Tage Angst ${ }^{12}$ (Bayern 2), Zündstoff ${ }^{13}$ von republik.ch (hochdeutsche Fassung: Knonau reichts) und viele Geschichten aus der Reihe Einhundert ${ }^{14}$ von Deutschlandfunk Nova. Neben diesen narrativen Podcasts setzen auch aktuelle News- und Interview-Formate Storytelling-Techniken ein. Darunter etwa Der Tag ${ }^{15}$ vom Deutschlandfunk, die funk-Produktion Deutschland30oo ${ }^{16}$, NZZ Akzent ${ }^{17}$ und auch das Coronavirus-Update ${ }^{18}$ (NDR Info). Bei letzterem ist ein zentraler Faktor die Persönlichkeit von Virologe Christian Drosten, der mit seiner unaufgeregten, reflektierten und zugewandten Art im Storytelling-Sinn als ein Charakter zu verstehen ist, zu dem man eine emotionale Verbindung aufbauen kann.

1 Zu meinem Hintergrund: Als Produzent, Autor und Regisseur habe ich mich auf Storytelling und komplexe Darstellungsformen in Podcasts und Radio spezialisiert. Neben meiner Aufgabe als Chefautor des WDR-Podcasts StoryQuarks begleite ich unter anderem als Story-Consultant die Entwicklung neuer Formate. Darüber hinaus moderiere ich bei Deutschlandfunk Nova den Podcast Deep Talk.

2 https://www.thisamericanlife.org/ (Zugriff am 10.10.2020).

3 https://www.wnycstudios.org/podcasts/radiolab (Zugriff am 10.10.2020).

4 https://www.npr.org/podcasts/510307/invisibilia (Zugriff am 10.10.2020) (Zugriff am 10.10.2020)

5 https://stownpodcast.org/ (Zugriff am 10.10.2020)

6 https://serialpodcast.org/ (Zugriff am 10.10.2020)

7 https://www.ttfa.org/ (Zugriff am 10.10.2020).

8 http://loveandradio.org/ (Zugriff am 10.10.2020)

9 https://99percentinvisible.org/ (Zugriff am 10.10.2020).

10 https://www.audible.de/ep/Der-Moment-Original-Podcast (Zugriff am 10.10.2020)

11 https://www.ndr.de/nachrichten/info/podcast4576.html (Zugriff am 10.10.2020).

12 https://www.br.de/mediathek/podcast/4-tage-angst/812 (Zugriff am 10.10.2020).

13 https://zuendstoff.podigee.io/ (Zugriff am 10.10.2020).

$14 \mathrm{https}: / /$ www.deutschlandfunknova.de/einhundert (Zugriff am 10.10.2020).

15 https://www.deutschlandfunk.de/deutschlandfunk-der-tag.3414.de.html (Zugriff am 10.10.2020).

16 https://audiothek.ardmediathek.de/programsets/66261430 (Zugriff am 10.10.2020).

17 https://www.nzz.ch/podcast/akzent (Zugriff am 10.10.2020)

18 https://www.ndr.de/nachrichten/info/podcast4684.html (Zugriff am 10.10.2020). 
Doch es fehlt gerade im journalistischen Feld - das ist zumindest mein Eindruck aus der praktischen (und vermittelnden) Arbeit - in Summe noch immer so etwas wie ein Storytelling-Grundverständnis, ein bewusster und sicherer Umgang mit den entsprechenden Techniken. Das beginnt schon beim Begriff: Das Wort ,Storytelling' sorgt häufig eher für Irritation oder sogar Ablehnung, vor allem unter Journalist:innen. Zu sehr hört es sich offenbar nach Fakten erfinden und Interviews verfälschen an. Der Tenor: Geschichten seien eben Geschichten und damit Fiktion, kein Journalismus. Dies offenbart ein grundlegendes Missverständnis darüber, was „Storytelling eigentlich meint: dramaturgische Techniken einsetzen, um eine Geschichte zu erzählen" (Preger 2019: 15, Herv.i.O.).

Eine Geschichte ist dabei dramaturgisch klassischerweise definiert als eine szenische Erzählung, in der eine zentrale Figur ein Ziel verfolgt und auf Hindernisse stößt. ${ }^{19}$ Diese Denkweise wird zwar in fiktionalen Formaten häufiger verwendet als in dokumentarischen, aber das bedeutet nicht, dass Storytelling auf fiktionales Erzählen festgelegt ist. Denn gerade Storytelling hat das Potenzial, Fakten erfahrbar zu machen, was letztlich eine zentrale Aufgabe von Journalismus darstellt. Das heißt, wenn es um journalistische Podcasts geht, muss dabei natürlich derselbe Wahrheitsanspruch gelten wie für alle anderen journalistischen Formate.

Die beschriebene Haltung vieler Journalist:innen offenbart also ein mangelhaftes Verständnis davon, was Storytelling meint und zu leisten im Stande ist. Schwierig wird es, wenn fehlendes Verständnis und sogar Ablehnung mit in die Podcast-Entwicklung genommen werden. Dann passiert nämlich genau das, was der oben erwähnte Ausruf der Seminarteilnehmerin umschreibt: Es wird das kopiert, was verstanden wird (häufig nach US-amerikanischen Vorbildern). Die Fragen für neue Produktionen lauten dann: Gibt es einen Host oder nicht? Wie lang soll etwas sein (entweder die typische Fahrtlänge zur Arbeit oder doch lieber so lang bis zwar alles gesagt wurde, aber noch nicht von jedem)? Welche Töne oder Situationen sind zu einem Thema vorstellbar (im Sinne von: Man sammelt schon mal Zutaten, ohne dass klar ist, was für ein $\mathrm{Ge}-$ richt eigentlich entstehen soll)? Und: Was machen andere? Genau das sollten wir dann auch machen (Stichwort: True Crime oder Corona).

Damit keine Missverständnisse entstehen: das sind teils wichtige Fragen. Sie sind aber zu Beginn einer Formatentwicklung oder am Anfang der Recherche zu einer Geschichte nur wenig hilfreich. Antworten auf diese Fragen muss man finden - aber erst, wenn klar ist, was man umsetzen oder entwickeln will. Der Ruf nach Storytelling wird indes meist dann laut, wenn es darum geht, die Produktion noch irgendwie , aufzupeppen'. Doch Storytelling ist kein Zauberstab, sondern eine Kulturtechnik des Erzählens, die sich durch den ganzen Entwicklungsprozess ziehen sollte, damit sie ihre Stärken entfalten kann. Aus der Praxisperspektive gibt es dabei ein paar Aspekte, die einerseits für ein tieferge-

19 Diese Art Geschichten, die von Protagonist:innen vorangetrieben werden, findet sich in fast allen klassischen Strukturmodellen wieder, wie etwa Akt-Struktur, Plot Point-Paradigma oder ,Heldenreise'. In meiner Arbeit habe ich weitere dramaturgische Modelle entwickelt, die Storytelling-Techniken auch in Geschichten ohne eindeutige Protagonist:innen einsetzen (vgl. Preger 2019). 
hendes Verständnis von Storytelling-Techniken in journalistischen Podcasts wichtig sind. Und deren Einsatz andererseits mit einigen Herausforderungen für die journalistische Arbeit verbunden ist - und darum soll es im Folgenden gehen.

\section{Podcast-Storys finden und strukturieren}

Wenn Storytelling grundsätzlich die Frage danach stellt, wie Fakten erfahrbar gemacht werden, dann ist damit auch das Ziel verbunden, Hörer:innen ein Erlebnis zu liefern - sie sollen die Bedeutung der Fakten spüren und nacherleben können - und sie gleichzeitig an die Geschichte zu binden. Handwerklich steht folglich die Frage im Mittelpunkt, wie man es schaffen kann, dass Menschen von Anfang bis Ende zuhören. Storytelling eignet sich dabei besonders für komplexe Stoffe, denn es lebt von Nuancen, Entwicklungen und Differenzierung.

Typische Storytelling-Fragen zu Beginn lauten daher: Was ist überhaupt eine gute Story -gerade aus journalistischer Sicht, die sich auf die Realität richtet? Oder bezogen auf einen neuen Podcast: Was ist das Alleinstellungsmerkmal? Irgendwann im Entwicklungsprozess sollte man darauf eine klare Antwort finden. Denn je klarer ist, was entstehen soll, desto mehr Ressourcen können darauf verwandt werden. Im Umkehrschluss: Je unklarer es ist (und möglicherweise je größer ein Team), desto eher wird in verschiedene Richtungen entwickelt und Energie geht verloren. Deswegen ist eines der zentralsten Ziele von Storytelling, Klarheit darüber zu erreichen, welche Geschichte erzählt werden soll. Und hier lauert schon die erste Gefahr für Missverständnisse: Das bedeutet nicht, dass Journalist:innen vorher festlegen, wie eine Geschichte ablaufen wird, wer wann etwas sagt oder wie Fakten auszusehen haben. Vielmehr meint Klarheit im Kontext von journalistischem Storytelling, benennen zu können, welche Geschichte man erzählen will.

Um das an einem Beispiel zu verdeutlichen: Als Stephan Beuting und ich im Podcast Der Anhalter ${ }^{20}$ die Geschichte des Obdachlosen Heinrich Kurzrock nachgezeichnet haben, haben wir zunächst unsere journalistischen Hausaufgaben erledigt und umfassend verschiedene Aspekte recherchiert: Missbrauch in Kinder- und Jugendpsychiatrien, Entschädigungspraxis, Lebensabschnitte der Beteiligten etc. Aus diesem gesamten Material wurde die Geschichte extrahiert. Dazu haben wir in einem ersten Schritt alle inhaltlichen Facetten in einer Art Mind-Map zusammengefasst, samt Gruppierung von zusammengehörigen Aspekten, Verbindungslinien, möglichen akustischen Umsetzungen usw. Hier hätten wir einen Punkt machen und die Folgen thematisch logisch sortieren können. Das hätte aber bei weitem nicht die Spannung entwickelt, die in dieser Geschichte enthalten ist. Also haben

$20 \mathrm{Im}$ Zentrum der Geschichte steht der Obdachlose Heinrich Kurzrock, der als Kind in einer Psychiatrie missbraucht wurde. Der daraus entstandene Doku-Podcast Der Anhalter wurde 2016 (fünf Teile) sowie 2019 (sechster Teil, Epilog) zuerst beim WDR veröffentlicht und erhielt u.a. 2017 den Deutschen Sozialpreis (vgl. https://www1.wdr.de/radio/wdr5/sendungen/tiefenblick/der-anhalter-dokuserie-100.html; Zugriff am 10.10.2020). 
wir das Material in einem zweiten Schritt nach dramaturgischen Gesichtspunkten noch einmal neu geordnet, in etwa nach folgendem Schema: Akt 1) Einstiegs-Szene, daraus Herleitung der Leitfrage oder zentrales Ziel für die jeweilige Folge; Akt 2) Versuch, dem Ziel näher zu kommen bzw. die Frage zu beantworten; Akt 3) Höhepunkt der Folge, also Beantwortung der Leitfrage (wenn möglich). Dabei sollte sich im Idealfall aus dem Höhepunkt der einen Folge eine weitere Frage ergeben (,Cliffhanger'), die dann in der nächsten Folge beantwortet wird. Außerdem sollte jede Folge der Beantwortung der zentralen Frage der gesamten Serie ein Stück näherkommen.

Lautet die dramaturgische Frage also grob, ob es so etwas wie Gerechtigkeit für Hauptfigur Heinrich geben kann, dann bringt uns die erste Folge mit der Frage, ob wir Heinrich überhaupt finden, auch dieser übergeordneten Leitfrage ein Stück näher. Bis es schließlich zum Höhepunkt der Serie kommt: der Konfrontation mit den Menschen, die am ehesten für das Leid von damals verantwortlich gemacht werden können. Für jede Folge gab es also Klarheit darüber, welche Geschichte wir erzählen, welchen Erzählbogen wir spannen wollten - ohne dass damit der Verlauf der Einzelfolgen und der Serie insgesamt schon in Stein gemeißelt war. Wir hatten zu diesem Zeitpunkt zwar bereits einige Aufnahmen während der Recherche gemacht, aber die entscheidenden Begegnungen standen uns noch bevor.

Was bei dieser Vorgehensweise hilft, ist ein Erzählsatz. Er besteht aus den zentralen Figuren der jeweiligen Folge, ihrer Motivation, ihrem Ziel und den Hindernissen auf dem Weg zu diesem Ziel (Preger 2019: 33ff.). Für die erste Anhalter-Folge (Beuting/Preger 2016a) lautet der Erzählsatz also etwa: Stephan und Sven wollen Heinrich finden, weil sie neugierig sind, eine Geschichte wittern, Heinrich interessant finden. Dafür müssen sie aber mehrere Hindernisse überwinden, u.a. Heinrichs Namen überprüfen, ihn wiederfinden, Teile seiner Geschichte verifizieren und schließlich zu ihm reisen.

Das Beispiel zeigt: Auch faktenbasierte, aufwändig recherchierte journalistische Podcasts lassen sich in Form eines Ablaufs, Storyboards oder Plotentwurfs strukturieren, die Inhalte also in eine dramaturgisch interessante Folge bringen - ohne dabei Ansprüche an journalistische Standards einzubüßen. Auf diesem Weg lässt sich zum einen Spannung erzeugen, die dafür sorgt, dass Hörer:innen der Geschichte bis zum Ende folgen. Zum anderen ergibt sich so die Möglichkeit, relevante Hintergrundinformationen in die Geschichte einzubauen, die aus journalistischer Perspektive wichtig sind. Dafür eignen sich bei weitem nicht nur Geschichten, die von Protagonist:innen vorangetrieben werden und bei denen sich die Spannung häufig aus der Frage ergibt, ob die Figur ihr Ziel erreichen wird. Auch abstraktere Stoffe sind vorstellbar, immer vorausgesetzt, die Themen lassen sich narrativ darstellen, also durch Szenen, die das Rückgrat einer jeden Geschichte bilden. Nur so kann eine sich entwickelnde Handlung - sprich: der Plot - entstehen.

Dieses Vorgehen, einen Plot zu entwerfen, eignet sich vor allem, wenn man an einer Geschichte arbeitet. Unabhängig davon, ob es sich um einen Einteiler oder eine ganze Serie handelt. Und hier lauert tatsächlich eine Gefahr: Spitzt 
man die Geschichte vielleicht zu sehr zu? Oder lässt man Details weg, die die Eindeutigkeit oder die Einheit der Geschichte gefährden würden?

Um dieses Risiko der Verkürzung zu vermeiden, ist es wichtig, die journalistische Mind-Map als eine Art Korrektiv immer griffbereit zu haben. Sie dient nach dem Erstellen des Plots zur Überprüfung, ob relevante Argumente genannt wurden und alle wichtigen Fakten enthalten sind. Werden hier Dinge weggelassen oder verfälscht, so können dafür kaum Storytelling-Techniken verantwortlich gemacht werden, sondern vielmehr mangelhaftes journalistisches Handwerk. Und warum sollte die Gefahr, Dinge wegzulassen oder nicht akkurat darzustellen, beim Storytelling größer sein als etwa in komprimierten Nachrichten oder tagesaktueller Berichterstattung?

Auch die Sorge, eine Geschichte zu glätten oder künstlich zu dramatisieren, widerspricht eher dem, was Storytelling eigentlich will: Bei vielen PodcastProduktionen sind es genau die Nuancen, Zwischen- oder Grautöne, die das Gefühl von Echtheit erzeugen oder noch verstärken. Noch dazu ist das reale Leben oft komplexer als eine aalglatte und vereinfachte Geschichte. Und für diese Form der Komplexität ist in Podcasts meist mehr Platz als in anderen journalistischen Formaten.

Etwas anders funktioniert die Fokusfindung unter Storytelling-Gesichtspunkten, wenn es um die Entwicklung eines neuen Podcasts geht. Dabei ist erst einmal egal, ob es sich um ein Interview- bzw. Gesprächsformat oder einen narrativen Podcast handelt. Die Ausgangsfrage ist immer die gleiche: Was soll der neue Podcast genau sein, was ist das Alleinstellungsmerkmal? Auch hier kann es helfen, dieses Merkmal in einem (möglichst konkret, aktiv und positiv formulierten) (Erzähl-)Satz auf den Punkt zu bringen und somit zu fokussieren.

Bei der Entwicklung von StoryQuarks ${ }^{21}$, einer 2019 gestarteten Podcast-Reihe des WDR, haben wir uns diesbezüglich von Radiolab und Invisibillia inspirieren lassen. Diese Formate liefern eine gute Idee davon, wie Host, Reporter:in und Gesamtsound klingen sollen. Gleichzeitig sind sie mit unterschiedlichen Alleinstellungsmerkmalen ausgestattet. Radiolab von WNYC Studios z.B. versteht sich der Selbstbeschreibung nach als ,, an investigation told through sounds and stories, and centers around one big idea. " 22 Drei Kernelemente werden hier deutlich: die Herangehensweise ( „an investigation”), die akustische Umsetzung („through sound and stories") und die Relevanz (, around one big idea”). Der NPR-Podcast Invisibilia ${ }^{23}$ wiederum versteht sich als „narrative journey through the invisible forces affecting human behavior" (Nuzum 2019: 33) so beschreibt es Eric Nuzum, der maßgeblich an der Entwicklung von Invisibilia beteiligt war. Für ihn ist die Grundidee, das Alleinstellungsmerkmal, der erste wichtige Schritt: „Describe your idea in no more than ten words, and do so in a way that describes nothing else in the world" (ebd.: 32).

Nuzum steht mit dieser Denkweise in der Tradition US-amerikanischer Er-

21 https://www.quarks.de/storyquarks/ (Zugriff am 10.10.2020).

22 https://www.kqed.org/radio/program/radiolab (Zugriff am 10.10.2020).

23 https://www.npr.org/podcasts/510307/invisibilia (Zugriff am 10.10.2020). 
zählformate, vor allem aus Hollywood. Blake Snyder beispielsweise arbeitet in Save the Cat, seinem Standardlehrbuch zum Drehbuchschreiben, mit den Begriffen ,Logline' oder ,Oneliner' (vgl. Snyder 2005: 4). Dahinter steckt eine ähnliche Idee, nämlich die Geschichte oder das Alleinstellungsmerkmal eines Projektes bzw. Produkts in einem Satz zusammenzufassen. Mit einer adaptierten Fassung arbeiten auch HowSound-Host Rob Rosenthal oder GimletGründer Alex Blumberg für ihre Podcasts - ihre Arbeitsweisen werden in der Graphic Novel Out on the Wire von Jessica Abel (2015: 52, 56f.) beschrieben. ${ }^{24}$

Das so formulierte Alleinstellungsmerkmal ist darüber hinaus eine Art Erzählversprechen, das der Podcast This American Life schon im Titel trägt: Es geht in jeder Folge um eine Facette des US-amerikanischen Zusammenlebens. Bei StoryQuarks erwähnen wir in der Formatbeschreibung ebenfalls eine Art Alleinstellungsmerkmal oder Kernelement für den Podcast: „Unbekanntes entdecken, die Welt verbessern oder herausfinden, wer wir sind - das geht oft nur mit Wissenschaft. StoryQuarks erzählt Wissens-Geschichten, die kraftvoll, komplex und manchmal kurios sind. Es sind Geschichten, die uns berühren und Euch hoffentlich auch. " ${ }^{25}$ Diese Beschreibung enthält etwas mehr als die von Nuzum geforderten zehn Wörter und ist möglicherweise nicht spezifisch genug. Doch die für uns als Macher:innen wesentlichen Elemente sind darin enthalten: Jede Folge behandelt eine echte Geschichte. Es soll außerdem einen Wissenskern geben, der eine entscheidende Rolle in der Geschichte spielt. Auf diese Weise sollen Fakten erfahrbar werden, wobei unsere Haltung erkennbar sein darf (was allerdings nicht heißt, dass journalistische Distanz verloren geht). Neben dieser strukturellen Story-Arbeit gibt es einzelne narrative Storytelling-Techniken, die dann wiederum beim Erzählen der Geschichte helfen - um einige der zentralsten geht es im Folgenden.

\section{Eine Podcast-Geschichte erzählen: Hilfreiche Storytelling-Techniken}

\subsection{Szenisches Erzählen}

Sowohl beim Anhalter (einer abgeschlossenen Serie) als auch bei StoryQuarks, einem fortlaufenden Format mit in sich meist abgeschlossenen Episoden, ist es wichtig, die Geschichte erfahrbar und nachvollziehbar zu gestalten. Szenen sind dabei das zentrale Element der Storys. Sie sind definiert als MiniStory, also als eine Situation, in der jemand etwas erreichen möchte, aber auf Hindernisse stößt (Preger 2019: 173ff.). Szenen erfordern wiederum prozessuales oder sequenzielles Erzählen, also das Erzählen entlang des Erkenntnisprozesses (weshalb viele Geschichten immer noch chronologisch erzählt werden) - im Gegensatz zu ergebnisorientierten Formaten wie z.B. Nachrichten. Gemeint ist damit allerdings nicht, jeden Schritt der eigenen Recherche nachzuerzählen. Auch wenn es in Zeiten, in denen Journalismus in der Kritik steht,

24 Zu dieser Debatte hörenswert ist die Episode „Wie man von den Besten lernt. Und trotzdem sein eigenes Ding macht" des Podcasts Frequenz vom Berliner Label Viertausendhertz (Efert et al. 2020).

25 https://audiothek.ardmediathek.de/programsets/66294074 (Zugriff am 10.10.2020). 
sicherlich angebracht ist, Transparenz über den Arbeitsprozess herzustellen. Aber es gibt so etwas wie ,Über-Transparenz' . Es ist beispielsweise dramaturgisch nicht wichtig, jedes einzelne Telefonat aufzunehmen, was in Deutschland ohne Einwilligung meines Gegenübers ohnehin nicht zulässig ist. Nicht nur dramaturgisch zeigen sich hier Grenzbereiche: Wie etwa sind Situationen zu bewerten, bei denen Reporter:innen ganze Telefonate von Anfang an mitschneiden und diese so auch gesendet werden - und bei denen es so klingt, als sei das Telefonat tatsächlich der erste Kontakt? Wird hier einfach die Aufnahme gestartet und nachher um Erlaubnis gefragt? Was juristisch, vorsichtig ausgedrückt, schwierig wäre. Oder gab es vorher Absprachen? Und wenn das der Fall ist: Wie echt ist der angeblich so authentische Eindruck dann noch? Ist dies nicht vielmehr eine behauptete Transparenz - und geht es bei dieser Form der Pseudotransparenz nicht eher um eine Selbstinszenierung? Unabhängig davon, wie man ein solches Vorgehen bewertet: Grundlegend muss die nacherzählte Recherche - und das gilt für jegliches Material, das eingebunden wird - der Geschichte dienen und diese vorantreiben, also eine echte Szene für die erzählte Story darstellen, sonst spielt sie keine Rolle.

Jetzt könnte man einwenden: normale Gespräche mäandern doch auch. Wenn eine Geschichte ein Gespräch oder eine Wendung enthält, die die Erzählung nicht weiterbringt, dann bildet dies doch Realität ab. Das mag so sein - auch wenn dahinter oft eher der Wunsch steckt, nicht noch mühselig kürzen zu müssen. Entscheidend aus narrativer Perspektive ist allerdings, ob die zusätzliche Gesprächsschleife noch etwas anderes enthüllt, z.B. ein wichtiger Fakt zur Sprache kommt. Oder die Art und Weise, wie jemand sich unterhält, zeigt etwas in Bezug auf den Charakter der Person. Solche Elemente können für die Geschichte wichtig sein. Behält man allerdings zu viele Passagen, die keine weitere Bedeutung haben, dann ist die Gefahr groß, Langeweile zu produzieren. Nur weil es möglich ist, in Podcasts lange, komplexe Geschichten zu erzählen, heißt das noch nicht, dass jede Geschichte diese Länge auch trägt. Im Gegenteil: Gerade dicht erzählte Geschichten entfalten einen besonderen Sog. Wird eine Geschichte hingegen noch mit eigener, unnötiger Reflexion aufgeladen (auch hier wird der erzählerische Stil von Serial-Reporterin Sarah Koenig häufig falsch kopiert), dann besteht die Gefahr, die Geschichte künstlich zu dramatisieren. Oder sie mit einer ,bigger idea' (Preger 2019: 28ff.), also einer zentralen Idee, zu versehen, die überhaupt nicht in der Geschichte angelegt ist. Nicht jeder Nachbarschaftsstreit ist eben ein Abbild des generellen gesellschaftlichen Umgangs miteinander. Die angemessene Länge und inhaltliche Dichte für eine Geschichte zu finden, ist eine journalistische Qualität und Herausforderung (Preger 2019: 96ff.) und hängt eben maßgeblich damit zusammen, wie viele Szenen man für eine Geschichte findet.

\subsection{Erzählhaltung: Sokratisch, nicht naiv}

Das bringt uns zur nächsten großen Storytelling-Gefahr, der vor allem Reporter:innen erliegen können: der ,Naivitäts-Falle'. Will ich den Rechercheprozess für Hörer:innen erfahrbar machen, dann kann es sehr verlockend sein, 
mich als besonders naiv darzustellen (Mead 2018). Grundsätzlich eine gute Idee: Einerseits wird bei dieser Vorgehensweise nachvollziehbar, wie sich eine Geschichte entwickelt. Andererseits wirkt der oder die Reporter:in nicht wie ein:e allwissende:r Erzähler:in (was bei den Hörer:innen mitunter zu Ablehnung führen kann).

Damit diese Storytelling-Technik der nichtwissenden oder ,sokratischen' Erzählhaltung glaubwürdig und damit gut eingesetzt werden kann, sind jedoch zwei Aspekte entscheidend: Erstens muss der dargestellte Wissensstand tatsächlich dem Wissensstand entsprechen, den man als Reporter:in in der jeweiligen Situation gehabt hat. Die Situation muss also wahrhaftig und authentisch abgebildet werden. Dies gelingt wahrscheinlich eher, je normaler sich die Situation (z.B. auch in Interviews) für Gesprächspartner:innen anfühlt. Und zweitens sollte auch hier die Situation die Geschichte wirklich vorantreiben.

Das bedeutet aber nicht, dass der Ausgang einer Szene vorher schon festgelegt oder nur dann berichtet wird, wenn es in die eigene Planung passt. Sowohl beim Anhalter als auch bei StoryQuarks legen wir viel Wert darauf, dass wir wissen, worauf eine Geschichte hinausläuft, welches Ereignis und welche Szene also am Ende steht. Dabei ist aber nicht entscheidend, was bei dieser Szene herauskommt - das können wir als Journalist:innen nicht vorhersagen. Stattdessen sehen wir uns verpflichtet, die Situation mit genügend Offenheit zu begleiten, wie es etwa in der finalen Konfrontation mit den verantwortlichen Personen in der fünften Anhalter-Folge „Zahltag“26 der Fall war (vgl. Beuting/Preger 2016b). Genau solche Situationen oder Szenen lassen dann auch eine andere zentrale Storytelling-Stärke von Podcasts hervortreten.

\subsection{Erzählweise: Nah und respektvoll}

Damit sind wir bei den größten Stärken von Audio-Storytelling: Nähe und Intimität. Dabei geht es nicht nur um den direkten Charakter des Mediums, der z.B. durch das Hören über Kopfhörer entstehen kann. Unter StorytellingGesichtspunkten ist wohl der stärkste Faktor, dass Menschen an die Geschichte gebunden werden, weil sie mitdenken und -fühlen. So können die Geräusche eines Krankenhausflures schon ausreichen, um ganze Szenen im Kopf der Hörer:innen hervorzurufen. In solche Szenen eingebettet sind dann im Idealfall Dialoge, die die Erzählung vorantreiben - gerade Stimme bzw. Dialog enthüllt Charakter und bindet Hörer:innen an die Figuren einer Geschichte. Die finale Szene der dritten Folge von StoryQuarks ( „Chance in Phase $2^{(127}$ ) spielt sich genau auf einem solchen Krankenhausflur ab. Wir warten zusammen mit der Familie, die wir im Verlauf der Folge kennengelernt haben, auf einen wichtigen Befund. Und dann kommt der Arzt um die Ecke... (vgl. Preger et al. 2019). In nur wenigen Sekunden Dialog entfalten sich hier viele Emotionen: Sorge, Anteilnahme, Erschöpfung. Diese Emotionen entstehen beim Hören, ohne dass sie direkt benannt werden müssen. Inszenatorisch

26 https://www1.wdr.de/mediathek/audio/wdr5/wdr5-tiefenblick/der-anhalter/audio-der-anhalter-zahltag-102.html (Zugriff am 10.10.2020).

27 https://www.quarks.de/podcast/storyquarks-folge-3-chance-in-phase-2/ (Zugriff am 10.10.2020). 
folgt die Szene damit dem Storytelling-Grundsatz: ,show, not tell'.

Entscheidend bei solchen Aufnahmen ist das Fingerspitzengefühl als Reporter:in. Die oben geschilderte Szene entwickelt ihre Wirkung aus mehreren Gründen. Sie hält einerseits respektvolle Distanz: Sie emotionalisiert nicht künstlich, sondern gibt den Personen Raum zum Handeln, sie dokumentiert also authentisch. Andererseits steht die Szene am Ende einer langen Geschichte (hier etwa 35 Minuten). Als Hörer:in haben wir die Personen bereits ausführlich kennengelernt. Diese inhaltliche Tiefe lässt sich kaum in zwei oder drei Minuten erzeugen. Und das ist einer der großen narrativen Unterschiede zwischen aktuellem Radiostück und Podcast: In einem kurzen Beitrag wäre die Gefahr groß, als Reporter:in oder Moderator:in noch mehr Drama aufbauen zu wollen. Außerdem müsste man sehr viel Kontext erklären, damit man die Situation versteht. Sie könnte so nicht aus sich selbst heraus Kraft entfalten. In einem langen Format wie dem Podcast hingegen ist Platz für schrittweise Entwicklung, Differenzierung, Tiefe und das Entstehen einer (gefühlten) Nähe zu den Protagonist:innen.

Diese Art von Nähe bringt jedoch Gefahren mit sich. Die größte ist wohl, dass man als Reporter:in unhinterfragt das Narrativ der Protagonist:innen reproduziert. Was ich damit meine: Hat man es als Journalist:in geschafft, Nähe zu einer Person aufzubauen, so fällt es mitunter schwer, wieder auf die journalistisch gebotene Distanz zu gehen. Also einen Schritt zurückzutreten und die Geschichte nüchtern zu betrachten, mit bewusst skeptischem Blick: Hat ein:e Protagonist:in möglicherweise einen eigenen Anteil an bestimmten Entwicklungen? Gibt es Aspekte, die man berechtigterweise kritisieren kann? Sind Widersprüche oder Lücken in der Erzählung erkennbar (das ist gerade bei nacherzählten Szenen wichtig)?

Dieser Wechsel zwischen Nähe und Distanz ist ohnehin eine klassische journalistische Fähigkeit und gerade bei journalistischen Podcasts, in denen durch Storytelling Nähe und Intimität aufgebaut wird, eine wichtige Qualität. Handwerklich bedeutet das vor allem, auf Plausibilitäten und Widersprüche zu achten. Und die Aussagen - wie bei anderen journalistischen Formaten auch - so gut es geht zu belegen. Die spätere Distanzierung fällt erfahrungsgemäß auch dann leichter, wenn man von Anfang an mit offenen Karten spielt. Beim Anhalter haben wir z.B. frühzeitig mit Heinrich geklärt, dass wir seine Behauptungen nach journalistischen Maßstäben überprüfen werden. Storytelling braucht also neben einer guten Geschichte Verantwortung, Vertrauensarbeit und Transparenz unter den beteiligten Personen. Das bedeutet auch, gewisse (kommunikative und ethische) Grenzen einzuhalten.

In den vergangenen Jahren sind etliche Produktionen entstanden, die sich mit genau diesen Grenzen eher schwergetan haben. In einem New YorkerArtikel geht Rebecca Mead (2018) mit $S$-Town ${ }^{28}$, Serial ${ }^{29}$ und Missing Richard Simmons $^{30}$ auf drei sehr bekannte journalistische Podcastformate ein. In

28 https://stownpodcast.org/ (Zugriff am 10.10.2020).

29 https://serialpodcast.org/ (Zugriff am 10.10.2020).

30 https://www.topic.com/missing-richard-simmons (Zugriff am 10.10.2020). 
allen drei Produktionen ist der Fall jeweils etwas unterschiedlich gelagert, sie weisen aber zusammen auf eine Kernfrage hin: Wie tief dringe ich in das Privatleben von Personen ein und wie gehe ich damit bei der Veröffentlichung um? Host Sarah Koenig kann sich in der ersten Staffel von Serial schließlich mit Adnan Syed über genau diese Grenzen austauschen - ein sehr berührendes Telefonat. Diese Chance hat Host Brian Reed in S-Town nicht mehr. Die zentrale Figur seines Podcasts, John B. McLemore, hat sich das Leben genommen. Das erfahren die Hörer:innen am Ende der zweiten Folge. Reed entscheidet sich dennoch dazu, viele Details aus McLemores Leben zu veröffentlichen. Wiederum anders ist der Fall bei Missing Richard Simmons. Simmons hat sich u.a. als Fitnesstrainer in den USA einen Namen gemacht und in der Öffentlichkeit lange Zeit als extrovertierte Person präsentiert. 2014 zieht er sich aus dem öffentlichen Leben zurück. Reporter Dan Taberski macht sich schließlich auf die Suche nach Simmons. Der Podcast enthält etliche Andeutungen und Spekulationen über Simmons' Privatleben.

Für mich persönlich sind hier einerseits die rechtlichen Rahmenbedingungen entscheidend. Aber es gibt noch einen weiteren, weicheren Faktor, der etwas mit der Idee zu tun hat, für die Storytelling grundsätzlich steht. Storytelling versucht, Entwicklungsprozesse von Menschen abzubilden und Nuancen gerecht zu werden. Die Heldenreise von Joseph Campbell (1949) etwa ist nicht nur eine Metastruktur für Geschichten, sondern auch eine moralische Aussage: der Held findet sich, um der Gemeinschaft und einem höheren Ziel zu dienen. In diesem Sinne steht Storytelling auch für Respekt, Anstand und den transparenten Umgang miteinander. Diese Erzählhaltung spürt man Brian Reed in S-Town die ganze Serie über ab. Anders ist dies bei Missing Richard Simmons. Hier scheint die Jagd im Mittelpunkt zu stehen, weniger der Respekt vor der Person (vielleicht hat Richard Simmons gute und persönliche Gründe für sein Verhalten, die niemanden etwas angehen).

Auch eine der herausforderndsten Situationen in Der Anhalter verdeutlicht dieses Problem: Wir haben von Heinrich in der zweiten Folge den Auftrag erhalten, seine Familiengeschichte zu recherchieren. Wir wollen wissen, warum er überhaupt in die Kinder- und Jugendpsychiatrie gekommen ist. Wir finden einiges heraus, wahrscheinlich auch mehr als Henrich gedacht hat. Wir sind stolz, wollen ihm alles erzählen. Aber als wir uns mit ihm treffen, will er von seiner eigenen Familiengeschichte nichts mehr wissen. Wir müssen akzeptieren: Er hat ein Recht auf Nichtwissen. So stolz wir auf unsere Rechercheergebnisse auch sein mögen, sie kommen in der Serie nicht vor. Zum Glück haben wir die ganze Szene aufgezeichnet, sie ist später eine der längsten und wirkmächtigsten in der gesamten Serie. Denn sie verdeutlicht die Komplexität seines Charakters, Widersprüche und unseres Verhältnisses zu ihm - der transparente und erfahrbar gemachte Umgang mit der Entscheidung unseres Protagonisten wird zu einem wichtigen Bestandteil der Erzählung. Für das Medium Podcast bedeutet das: Gerade weil Audio so intim ist, braucht es keine künstliche Dramatisierung oder Pseudonähe. Zugleich muss die Geschichte immer ein angemessenes Abbild von Realität liefern, sonst hat es zumindest mit journalistischen Podcasts nicht viel zu tun. 


\subsection{Erzählton: Den richtigen Sound treffen}

Das bringt uns zu einem weiteren zentralen Story-Gestaltungselement: dem Sound. Das vielleicht auffälligste Mittel ist Musik. Wie sie wahrgenommen wird, geht zwar häufig weit auseinander, aber es gibt Kriterien für einen sinnvollen narrativen Musikeinsatz. Ein wichtiger Grundsatz aus meiner Sicht: Musik kommentiert immer. Sie verstärkt, widerspricht, deutet eine Situation aus oder gibt so etwas wie narrative Orientierung (Preger 2019: 248ff.). Ein eindrucksvolles Beispiel ist der Soundtrack von S-Town: Von Anfang an verortet die obskur-verspielte Musik die Geschichte als etwas abseitig der sonst üblichen Realität (was natürlich eine Deutung und Bewertung seitens der Produzent:innen darstellt).

Musik wird außerdem zur emotionalen Verstärkung eingesetzt, was aber mit Risiken besetzt ist. Wenn etwa die Musik eine ohnehin emotionale Situation noch zusätzlich auflädt, dann kann die Wirkung auch unfreiwillig komisch sein. Für meine Regie-Arbeit bei dokumentarischen Stoffen (wie bei Der Anhalter oder Zeitzeichen ${ }^{31}$ ) nutze ich Musik in realen Szenen eher selektiv, um eine Stimmung zu Beginn der Szene zu setzen oder eine bestimmte Stelle meist zum Ende der Szene - zu akzentuieren bzw. zu markieren. In Folge 11 von StoryQuarks („Hosen machen Leute “32) besucht Reporterin Cornelia Wegerhoff z.B. die Schafe, aus deren Wolle eine bestimmte Hose rekonstruiert werden soll. Den Einstieg in diese Szene haben wir mit einer etwas verträumten Musik markiert, die die Hörer:innen aufs Land mitnimmt. In die Musik legen wir die ersten Dialog-Sequenzen, die zentralen Infos zum Ort und ein paar Originalaufnahmen der Schafe (vgl. Wegerhoff et al. 2019). Damit ist die Stimmung gesetzt, die Musik endet. Nun kann sich die reale Szene ohne $\mathrm{Mu}-$ sik weiterentwickeln, sonst wird es sehr schnell kitschig. Innerhalb der Szenen kommt damit weniger Musik zum Einsatz. Sie taucht z.B. präsenter in Höhepunkten von Szenen auf, um besondere Momente nachklingen zu lassen. Wie in der oben geschilderten Szene aus dem Krankenhaus: Wir erfahren mit der Familie die Ergebnisse der Untersuchung und brauchen nun auch als Hörer:in einen Moment, um durchzuatmen und für uns zu sein. Dieser Raum wird mit Musik geschaffen.

Musik als narratives Gestaltungsmittel ist zumeist eindeutig erkennbar, auch wenn man beim Hören im Sinne der Immersion nicht darüber nachdenken sollte. Etwas heikler kann es bei Gestaltungselementen werden, die für die Hörer:innen nicht oder kaum wahrnehmbar sind. Noch eher unproblematisch sind Techniken, die dabei helfen, sich besser auf den vorhandenen Inhalt zu konzentrieren. Dazu zählt etwa das Säubern von Sprachaufnahmen (ohne allerdings Sprachduktus oder Sprecheigenheiten zu verändern) oder die dezente akustische Aufbereitung, indem man z.B. das tiefe Brummen eines Kühlschranks entfernt. Merkt man im Gespräch allerdings, dass eine Person durch den brummenden Kühlschrank genervt ist, bleibt das Brummen natürlich drin. Das Ziel ist also nicht, einen besonders ,cleanen' Sound zu

31 https://www1.wdr.de/mediathek/audio/zeitzeichen/index.html (Zugriff am 10.10.2020).

32 https://www.quarks.de/podcast/storyquarks-folge-11/ (Zugriff am 10.10.2020). 
produzieren, sondern einen Sound zu gestalten, der gut ,hörbar' ist und zugleich die Realität angemessen akustisch abbildet. Das gilt selbst für fiktionale Podcastproduktionen wie Gimlet Media's Homecoming ${ }^{33}$ oder Limetown ${ }^{34}$ von Two-Up Productions, die anstelle eines sauberen Studiosounds teilweise auf realistischen, ,dreckigen' Dokuklang zurückgreifen, um ihre Geschichten zu erzählen.

Um Realität auch wirklich , authentisch' abzubilden, sollte man bei der Inszenierung von realen Szenen bevorzugt mit akustischen Mitteln arbeiten, die tatsächlich aus der Aufnahmesituation stammen. Manchmal ist das aber nicht möglich, weil gewisse Aufnahmen fehlen oder es psycho-akustisch wenig Sinn ergibt. Dann steht man vor der Frage, wie viel (und welche) Atmo, Geräusche oder Effekte (z.B. aus Sound Datenbanken oder Archiven) man einsetzt. Besonders bei der Inszenierung rekonstruierter, also z.B. nacherzählter oder erinnerter Szenen, ist die Gestaltung von immersivem Sound eine Herausforderung. In Folge 2 von StoryQuarks („Spendenquittung ${ }^{(135}$ ) beispielsweise ist der Einstieg eine rekonstruierte Szene. Die Protagonistin kommt am Flughafen an, wo sie von ihrer Mutter abgeholt wird. Die Mutter offenbart ihr, dass die beiden über etwas Wichtiges reden müssen, sagt aber nicht, worum es geht (vgl. Pache et al. 2019). Es ist dramaturgisch gesprochen das auslösende Ereignis der Geschichte. Eine Szene, die mehr erzählerische Kraft und Immersion entwickelt, wenn man sie sich vorstellen kann. Deshalb haben wir sie mit Musik, ,Atmo' und Geräuschen inszeniert und somit besser erfahrbar gemacht. Gerade der Musik kommt hier eine entscheidende Rolle zu: Anders als bei Szenen , aus erster Hand' bindet sie die gesamte Szene zusammen und macht es zugleich möglich, gezielt Akzente zu setzen, um den Erzählrhythmus zu gestalten.

Insgesamt stellt also auch die akustische Inszenierung ein wichtiges narratives Element dar. Gerade in journalistischen Podcasts sollte ein Ziel des Einsatzes von Storytelling-Soundtechniken sein, ein angemessenes, ,authentisches' Abbild von Realität erfahrbar zu machen. Eine besondere Herausforderung an Regie und Sound-Design stellen dabei Rekonstruktionen wie z.B. erinnerte Szenen dar, die ein wichtiger narrativer Baustein sein können. Hier sollte die akustische Inszenierung transparent sein, um keinen falschen Eindruck bei den Hörer:innen zu erzeugen.

\section{Storytelling: Teil der Lösung}

Der Gesamteindruck einer Erzählung wird also einerseits von der Akustik bzw. Gesamt-Komposition getragen, andererseits (mindestens genauso wichtig) von der Erzählhaltung, die Hosts und/oder Reporter:innen mitbringen. Und hier gilt am ehesten: bescheidene Erzähler:innen sind gute Erzähler:innen. Emotionen werden ohnehin nicht erzeugt, indem man sie auf übertriebene

33 https://gimletmedia.com/shows/homecoming (Zugriff am 10.10.2020).

$34 \mathrm{https://twoupproductions.com/limetown/podcast} \mathrm{(Zugriff} \mathrm{am} \mathrm{10.10.2020).}$

35 https://www.quarks.de/podcast/storyquarks-folge-2-spendenquittung/ (Zugriff am 10.10.2020). 
und damit wenig authentische Art und Weise, vorlebt'. Einen weiteren entscheidenden Faktor bringt Eric Nuzum gut auf den Punkt, indem er feststellt "that there is a big difference between being spontaneous and sounding spontaneous" (Nuzum 2019: 59, Herv. i.O.). In Der Anhalter haben wir die Skripte noch sehr ausformuliert und zugleich versucht, uns schon beim Schreiben an unserem natürlichen Duktus zu orientieren (eher Schreiben fürs Erzählen als Schreiben fürs Hören). Bei StoryQuarks gehen wir noch einen Schritt weiter und haben u.a. als Team einen Improvisationskurs absolviert, um im Gespräch Gedanken authentisch neu zu entwickeln, auch wenn wir sie vorher kennen. Das bedeutet auch, dass es nicht darum geht, andere Hosts zu kopieren. Nicht Ira Glass, nicht Jad Abumrad oder Sarah Koenig. ${ }^{36}$ Denn Storytelling - und dieser Grundsatz passt wie kaum ein anderer zu Podcasts - bedeutet letztlich auch, die eigene Stimme zu finden.

Bei StoryQuarks heißt das: Wir probieren aus, schärfen - und entwickeln dann weiter. Dies geht nur auf Basis enger Zusammenarbeit, um ein gemeinsames Verständnis für die erzählerische und journalistische Haltung des Formates zu entwickeln. Dabei schließen sich Storytelling und klassisches, journalistisches Handwerk nicht aus, sondern gehen vielmehr Hand in Hand. Das erfordert eine enge Verzahnung vieler Rollen und Gewerke (Recherche, Redaktion, Fact Checking, Hosts, Dramaturgie, Sound Design, Social Media, Marketing etc.), die nicht erst am Ende einer Produktion stehen kann. Bei den bekanntesten journalistischen Storytelling-Podcasts wird das Gesamtprodukt daher oft von großen Teams getragen. Bei StoryQuarks besteht dieses Team aktuell aus rund 15 Menschen.

Akustisch ist in diesen Jahren viel in Bewegung, die Lust der Macher:innen am Experimentieren und Ausprobieren ist groß. Neue journalistische Podcasts entstehen, die auf Storytelling setzen, narrative Formate genauso wie Interviewproduktionen oder Mischformen. Diese Podcasts erreichen Hörer:innen, die möglicherweise mit dem klassischen linearen Radio (nur noch) wenig anfangen können - oder bisher kaum Zugang zu bestimmten Themen gefunden haben. Storytelling ersetzt dabei nicht, sondern ergänzt bisherige journalistische Darstellungsformen. Es schafft bei verantwortungsvollem Umgang die Möglichkeit, gerade komplexe Phänomene, Themen und Geschichten stärker in ihrer Ganzheit abzubilden. Sie erfahrbar zu machen und nicht nur schlaglichtartig (oftmals mit dem Fokus auf dem Negativen) zu beleuchten, Ambivalenzen und Widersprüche aufzuzeigen. Für die journalistische Vermittlung gesellschaftlich relevanter Themen ist dieses Potenzial erzählerischer Mittel - wenn sie kompetent eingesetzt werden - sehr wertvoll. Daher sollte Storytelling in Podcasts nicht als ,Zaubermittel', sondern als eine wichtige Technik des lösungsorientierten, konstruktiven Journalismus verstanden und ernst genommen werden.

36 Wie die drei arbeiten, kann man u.a. bei Biewen und Dilworth (2017) nachlesen. 


\section{Literatur}

Abel, J. (2015). Out on the Wire. The Storytelling Secrets of the New Masters of Radio. New York: Broadway Books.

Beuting, S., \& Preger, S. (2016a, Juni 5). Episode 1: Letzte Ausfahrt Zürich. Der Anhalter [Podcast]. Zugriff am 10.10.2020. Verfügbar unter: https://www1. wdr.de/mediathek/audio/wdr5/wdr5-tiefenblick/der-anhalter/audio-deranhalter--letzte-ausfahrt-zuerich-102.html

Beuting, S., \& Preger, S. (2016b, Juli 3). Episode 5: Zahltag. Der Anhalter [Podcast]. Zugriff am 10.10.2020. Verfügbar unter: https://www1.wdr. de/mediathek/audio/wdr5/wdr5-tiefenblick/der-anhalter/audio-deranhalter--zahltag-102.html

Biewen, J. \& Dilworth, A. (Hrsg.) (2017). Reality Radio. Telling True Stories in Sound. Durham: UNC Press.

Campbell, J. (1949). The Hero with a Thousand Faces. New York: Bollingen Foundation.

Efert, H., Semak, N., \& Conradi, C. (2020, Februar 2). Episode 63: Wie man von den Besten lernt. Und trotzdem sein eigenes Ding macht. Frequenz [Podcast]. Zugriff am 05.03.2020. Verfügbar unter: https://viertausendhertz.de/frq63/

Mead, R. (2018, November 19). How podcasts became a seductive - and sometimes slippery - mode of storytelling. New Yorker Magazine. Zugriff am 05.03.2020. Verfügbar unter: https://www.newyorker.com/magazine/ 2018/11/19/how-podcasts-became-a-seductive-and-sometimes-slipperymode-of-storytelling

Nuzum, E. (2019). Make Noise. A Creator's Guide to Podcasting and Great Audio Storytelling. New York: Workman Publishing.

Pache, V. et al. (2019, August 28). Episode 2: Spendenquittung. StoryQuarks [Podcast]. Zugriff am 10.10.2020. Verfügbar unter: https://www.quarks.de/ podcast/storyquarks-folge-2-spendenquittung/

Preger, A. et al. (2019, August 28). Episode 3: Chance in Phase 2. StoryQuarks [Podcast]. Zugriff am 10.10.2020. Verfügbar unter: https://www.quarks.de/ podcast/storyquarks-folge-3-chance-in-phase-2/

Preger, S. (2019). Geschichten erzählen. Storytelling für Radio und Podcast. Wiesbaden: Springer VS. https://doi.org/10.1007/978-3-658-23428-7

Snyder, B. (2005). Save the Cat. Sudio City: Michael Wiese Productions.

Wegerhoff, C. (2019, Dezember 31). Episode 11: Hosen machen Leute. StoryQuarks [Podcast]. Zugriff am 10.10.2020. Verfügbar unter: https://www.quarks. de/podcast/storyquarks-folge-11/ 